\title{
Deshumanización y legitimación de los conflictos armados en los videojuegos bélicos*
}

\section{Alejandro González-Vázquez ${ }^{1}$ Juan José Igartua Perosanz²}

Recibido: 26/09/2018

Aprobado por pares: 23/10/2018

Enviado a pares: 26/09/2018

Aceptado: 31/01/2019

DOI: 10.5294/pacla.2019.23.1.4

Para citar este artículo / to reference this article / para citar este artigo

González-Vázquez, A. e Igartua, J. J. (2020). Deshumanización y legitimación de los conflictos armados en los videojuegos bélicos. Palabra Clave, 23(1), e2314. D0I: https:// doi.org/10.5294/pacla.2019.23.1.4

\section{Resumen}

En este artículo se pretende recoger la representación o representaciones de la guerra presentes de manera predominante en los videojuegos de géneros bélicos. Para ello, se desarrolló un análisis de contenido sobre una muestra de 62 videojuegos elaborada siguiendo como criterio de muestreo el volumen de ventas o el número de descargas en España entre los años 2005 y 2015. Dicho análisis se articuló a partir de tres ejes conceptuales: a) los elementos legitimadores de los conflictos, b) los elementos deshumanizadores del enemigo y c) los elementos desindividualizadores de los jugadores. Los resultados muestran a) la prevalencia de un discurso legitimador del conflicto centrado en la defensa, sin motivaciones territoriales o de recursos y con ausencia de civiles durante la acción del videojuego, b) un predominio de enemigos de apariencia y comportamiento homogéneo, carentes de rasgos distintivos y representados de forma realista, y c) unos

\footnotetext{
* La presente investigación forma parte del siguiente trabajo de Tesis Doctoral: González-Vázquez, A. (2019). La Banalización de la Guerra en los Videojuegos Bélicos (Tesis doctoral). Universidad de Salamanca, Salamanca.

1 https://orcid.org/0000-0001-6485-031X. Universidad de Salamanca, España. alejandro.gonzalez@usal.es

2 https://orcid.org/0000-0002-9865-2714. Universidad de Salamanca, España. jigartua@usal.es
} 
personajes protagonistas caracterizados por su diseño predeterminado, su trasfondo heroico y su irreal invulnerabilidad al daño. La presente investigación, poseedora de carácter meramente descriptivo, permitie establecer una base empírica para estudios posteriores sobre la construcción de creencias y actitudes en los usuarios habituales de esta clase de ocio a través de la Teoría del Cultivo.

\section{Palabras clave (Fuente: tesauro de la Unesco)}

Videojuegos; análisis de contenido; contenido de programa; deshumanización; legitimación. 


\section{Dehumanization and Legitimization of Armed Conflicts in War Video Games*}

\section{Abstract}

This article collects predominant representation or representations of war in war video games. To this end, a content analysis is carried out on a sample of 62 video games, which was taken following the sampling criteria of volume of sales or number of downloads in Spain between 2005-2015. This analysis is based on three conceptual lines: a) conflict legitimizers; b) enemy dehumanizers; and c) player de-individualizers. Results show the prevalence of a defense-centered legitimizing discourse of the conflict with no territorial or resource motivations, the absence of civilians during the action of the game, a predominance of enemies with homogeneous appearance and behavior who lack distinctive features and are realistically represented, and some main characters with a default design, heroic background and unreal invulnerability. Relying on the cultivation theory, this descriptive work establishes an empirical basis for further studies on how regular users of this kind of leisure activity construct beliefs and attitudes.

\section{Keywords (Source: Unesco Thesaurus)}

Video games; content analysis; programme content; dehumanization; legitimacy.

* This article derives from González-Vázquez, A. (2019). La banalización de la guerra en los videojuegos bélicos (dissertation, Universidad de Salamanca, Salamanca, Spain). 


\section{Desumanização e legitimação dos conflitos armados nos videogames bélicos*}

\section{Resumo}

Este artigo trata da representação ou representações da guerra presentes de maneira predominante nos videogames de gêneros bélicos. Para isso, foi desenvolvida uma análise de conteúdo de 62 videogames que seguiu, como critério de amostra, o volume de vendas ou o número de downloads na Espanha entre 2005 e 2015. A análise está articulada a partir de três eixos conceituais: a) os elementos legitimadores dos conflitos, b) os elementos desumanizadores do inimigo e c) os elementos desindividualizadores dos jogadores. Os resultados mostram a prevalência de um discurso legitimador do conflito centralizado na defesa, sem motivações territoriais ou de recursos e com ausência de civis durante a ação do videogame, um predomínio de inimigos de aparência e de comportamento homogêneo, carentes de traços distintivos e representados de forma realista, e umas personagens protagonistas caracterizadas por seu desenho predeterminado, sua origem heroica e sua irreal invulnerabilidade ao dano. Este trabalho, de caráter descritivo, permitiu estabelecer uma base empírica para estudos posteriores sobre a construção de crenças e atitudes nos usuários desse tipo de lazer por meio da teoria do cultivo.

\section{Palavras-chave (Fonte: tesauro da Unesco)}

Jogos de vídeo; videogame; análise de conteúdo; conteúdo do programa; desumanização; legitimação.

* Este artigo é derivado da tese de doutorado de González-Vázquez (2019), intitulada "A banalização da guerra nos videogames bélicos" ("La banalización de la guerra en los videojuegos bélicos”), realizado na Universidad de Salamanca, Espanha. 


\section{Introducción}

Desde su popularización en la década de 1980, el estudio de los videojuegos y su posible influencia en sus usuarios ha sido un tema recurrente en el campo de la investigación psicosocial, especialmente en lo referente a la relación entre la exposición a contenidos violentos y el desarrollo de comportamientos agresivos (Dill \& Dill, 1999). A partir de perspectivas tanto cognitivistas como neoconductistas, durante las últimas décadas numerosos estudios se han centrado en esclarecer el papel que los videojuegos ejercen sobre los jugadores (De Miguel, 2012), sin haberse alcanzado aún un consenso académico entre aquellos autores que defienden la existencia real de efectos negativos en la conducta debido al consumo de esta clase de ocio (Dill \& Dill, 1999; Anderson, 2004; Anderson \& Dill, 2000) y aquellos que, en cambio, subrayan una ausencia de evidencia empírica suficiente que respalde estos efectos (Bensley y Van Eenwyk, 2001; De Miguel, 2012; Olson, 2004; Ferguson \& Kilburn, 2010; Griffiths, 1999).

Por tanto, no es extraño observar que cada vez más investigadores abogan por el estudio de los efectos de los videojuegos no solo desde el análisis de sus aspectos meramente conductuales y lúdicos (es decir, las diferentes acciones que puede realizar el jugador dentro del juego, como conducir, disparar un arma, robar, etc.), sino adoptando también un enfoque centrado en el aspecto más narrativo de esta clase de ocio, esto es, sus facetas más representacionales, culturales e ideológicas (Santorineos \& Dimitriadi, 2006), así como su posible repercusión no tanto a nivel conductual sino en la construcción de actitudes y creencias de los usuarios ante determinados fenómenos sociales (Kolek \& Šisler, 2017; Van Mierlo \& Van den Bulck, 2004; Williams, 2006). De este modo, en los últimos años, el campo de los game studies ha experimentado un especial desarrollo e incorporado al debate académico el uso de enfoques analíticos que buscan estudiar el discurso ideológico presente en cada uno de estos productos audiovisuales, es decir, el mensaje implícito en los videojuegos más allá de sus meras características técnicas (Pötzsch \& Hammond, 2016). En esta línea, los videojuegos bélicos son especialmente sensibles a esta clase de estudios, ya que estos juegos poseen un alto contenido violento (Marcano, 2014) y, a su vez, se caracterizan por tener una estructura narrativa a través de la cual 
el jugador se ve inmerso en la recreación de un conflicto armado, unas veces fantástico y otras basado en episodios históricos. En este último caso, esta recreación no se trata de un simple relato histórico, sino que a menudo deriva en una reinterpretación de los hechos en la que se proyectan determinados sistemas de valores e ideologías (Ford, 2016; Gagnon, 2010). Un buen ejemplo de ello es la omnipresencia del pensamiento colonial e imperialista en juegos de estrategia tales como la saga Civilization, que actúa como legitimador de las diferentes conductas que debe desarrollar el jugador (Ford, 2016; Šisler, 2008). El mismo fenómeno puede observarse aún con más intensidad en los videojuegos ambientados en las grandes guerras del siglo XX, cuanto frecuentemente se utiliza un planteamiento reduccionista de las causas del conflicto (Chapman, 2016), se obvian las consecuencias en la población civil (Rodríguez, 2014) y, en definitiva, se transmite una versión edulcorada y sesgada de la guerra (Gagnon, 2010; Hess, 2007). No obstante, quizá el punto de inflexión del auge de las implicaciones ideológicas de los videojuegos bélicos puede encontrarse en el cambio de siglo tras los atentados del 11 de septiembre en Nueva York y el comienzo de la llamada guerra contra el terror (Ouellette, 2008; Power, 2007; Sample, 2008). Numerosos autores coinciden en señalar la existencia de una retórica cada vez más recurrente que busca legitimar, implícita o explícitamente, determinadas posturas políticas y militares, que sitúa sistemáticamente al jugador en una representación del conflicto entendido como la lucha de un pueblo o nación que se defiende frente unos invasores de ideología o religión extremista cuyo objetivo es destruir la civilización, tanto en contextos realistas (Gagnon, 2010; Sample, 2008; Stamenković, Jaćević \& Wildfeuer, 2017) como ficticios (Lizardi, 2008; Voohrees, 2014).

\section{Deshumanización y desindividualización}

$\mathrm{El}$ aspecto representacional de los videojuegos bélicos, además, no se reduce al establecimiento de un discurso legitimador de los conflictos. Se traduce también en una determinada construcción del adversario basada habitualmente en su homogeneización, y así hace sencillo para el jugador establecer la diferencia entre aliados y enemigos (Bellmunt, 2012), y su deshumanización, y de esta manera facilita una suerte de desconexión moral que permita disfrutar del videojuego a los usuarios (Hartmann \& 
Vorderer, 2010; Klimmt, Schmid, Nosper, Hartmann \& Vorderer, 2006). A menudo, la representación del enemigo recurre al uso de estereotipos en los que los soldados del bando contrario portan la misma indumentaria y apariencia física (Šisler, 2008; Saleem \& Anderson, 2013), se comportan de forma gregaria y agresiva, $y$, a diferencia de la facción a la que pertenece el jugador, carecen de emociones y sentimientos nobles, como el honor o la piedad (Bellmunt, 2012).

Esta clase de elementos deshumanizadores no son fenómenos nuevos ni limitados al ámbito de los videojuegos; muy al contrario, han sido estudiados ampliamente por la psicología social en diferentes contextos y desde distintas perspectivas (Haslam, 2006; Rodríguez, 2014). Ya en la década de 1970 el archiconocido experimento de la Prisión de Stanford (Zimbardo, 2008) ponía de manifiesto el potente efecto de la imposición de uniformes y nombres degradantes en la aparición de conductas violentas a nivel intergrupal. Pese a que este estudio aún es objeto de controversia (Haslam y Reicher, 2004), existe evidencia suficiente para afirmar que la atribución de características extremadamente negativas al exogrupo (Bar-Tal, 1989), la percepción de ausencia de valores compartidos con el endogrupo (Struch \& Schwartz, 1989) y la negación de emociones e inteligencia en los miembros rivales (Leyens et al., 2001) suponen formas de deshumanización e infrahumanización capaces de legitimar por sí mismas la agresión hacia los "otros". Del mismo modo, Bandura (1999) señala la deshumanización como un proceso de exclusión moral que puede derivar en conductas violentas hacia el exogrupo, producido por mecanismos que incluyen el uso de eufemismos para describir la agresión; la minimización del daño causado; la atribución de culpa o el merecimiento a la víctima; la justificación, comparación y relativización de la conducta violenta; y la difusión de la responsabilidad. En relación con estos últimos mecanismos de desconexión, adquiere relevancia otro concepto clave en la psicología social del conflicto: la desindividualización. Dicho concepto, acuñado de nuevo por Zimbardo (1970) y desarrollado por Diener (1976), hace referencia al fenómeno de reducción de la percepción y evaluación de la propia identidad, su difusión en un grupo de pertenencia, su ocultamiento bajo otra identidad o el sentimiento de impunidad. Su importancia como facilitador 
de comportamientos agresivos ha sido comprobada en numerosos estudios (Postmes \& Spears, 1998; Rehm, Steinleitner \& Lilli, 1987; Silke, 2003) y ha dejado patente que en el grado en el que un individuo relativice su responsabilidad en las consecuencias de sus actos bajo un avatar (un uniforme, una máscara, una personalidad anónima) más probable será que realice conductas que de otro modo le resultarían inaceptables.

En los videojuegos bélicos, la conjunción de un discurso legitimador del conflicto con la existencia de elementos representacionales deshumanizadores y desindividualizadores puede dar lugar a una imagen de los conflictos bélicos sesgada, a través de la cual se transmita a los usuarios una visión banalizada de la guerra en la que se infravaloran las implicaciones y consecuencias terribles que acarrea para las sociedades y los individuos que en ella participan. Desde el punto de vista de la teoría del cultivo (Gerbner \& Gross, 1976), en la medida en la que una audiencia invierta mucho tiempo consumiendo un producto audiovisual más probable, será que crea que la realidad se asemeja a lo retratado en dicho producto. Así, la exposición a esta clase de videojuegos puede tener un efecto de cultivo en las actitudes y creencias de los usuarios (Van Mierlo. \& Van den Bulck, 2004; Williams, 2006), a la hora de legitimar y apoyar diferentes acciones bélicas, del mismo modo que ocurre con otros formatos audiovisuales ampliamente extendidos como el cine o la televisión (Igartua y Páez, 1997).

No obstante, para valorar adecuadamente en qué medida este fenómeno puede estar produciéndose en los videojugadores habituales, es necesario como paso previo delimitar hasta qué punto los videojuegos bélicos están efectivamente transmitiendo de forma generalizada esta clase de representaciones sesgadas de la guerra a través de la presencia de elementos legitimadores, deshumanizadores y desindividualizadores que, al darse de manera conjunta o individual, pueden contribuir a construir una determinada visión de los conflictos bélicos. Para ello, se hace necesario reforzar la evidencia empírica no solo mediante el estudio de caso (Ford, 2016; Gagnon, 2010; Hess, 2007; Rodríguez, 2014; Stamenković et al., 2017; Voorhees, 2014), sino también ampliando la investigación al análisis de los diferentes géneros en busca de aquellas herramientas o elementos representacionales 
que aparecen (o no) de manera recurrente y sistemática en los videojuegos, tal y como plantean los trabajos de Šisler (2008) y Chapman (2016), entre otros. No cabe duda de que la mayoría de dichos elementos pueden ser a su vez muy interesantes como objeto de análisis ludológico y no solo representacional; por ejemplo, en el caso de las instrucciones que se dan al jugador para alcanzar la victoria, se describe los aspectos más técnicos de la mecánica del juego, pero al mismo tiempo también se plantea una determinada representación de los objetivos a cumplir como característica principal y fin último de los conflictos bélicos. Es evidente, además, el potencial valor que puede tener el estudio exhaustivo del papel que dichos elementos realizan en la construcción de la narrativa general de cada obra audiovisual, como forma de abordar la identificación del discurso predominante en cada videojuego. Sin embargo, el objetivo de este artículo no es desarrollar un análisis lúdico-narratológico, es decir, el estudio de la interacción entre los personajes y el escenario, el ritmo narrativo, la figura del narrador, etc., ni su interconexión con las mecánicas de juego. Por el contrario, su intención es la realización de un análisis de contenido de los videojuegos bélicos más populares (concretamente en el mercado español) a través del cual identificar de manera puramente descriptiva la representación de la guerra más común en esta clase de producto audiovisual, mediante la observación de la frecuencia con la que aparecen determinados elementos representacionales.

\section{Metodología}

Para llevar a cabo los objetivos de este estudio, en primer lugar, se procedió a la elaboración de un libro de códigos que vertebrase el análisis de contenido. Dicho libro se construyó en torno a los tres ejes conceptuales antes mencionados: a) legitimación del conflicto, b) deshumanización del enemigo y c) desindividualización del jugador, subdivididos a su vez en diversas categorías que tomaron como referencia teórica los trabajos de los autores citados. De este modo, la legitimación del conflicto contempla el discurso legitimador y el realismo presentes en el juego, la deshumanización contempla los atributos físicos, el comportamiento y los mecanismos de desconexión moral vinculados a los enemigos y, por último, la desindividualización considera la construcción del avatar del jugador y la percep- 
ción de las consecuencias sufridas sobre dicho avatar tras participar en un enfrentamiento. Cada categoría está formada por entre dos y cuatro variables predominantemente dicotómicas (p. ej., caracterización del avatar: personalizada/por defecto) salvo determinadas excepciones (p. ej., naturaleza del enemigo: humana/humanoide/monstruosa), cuyo objetivo es codificar de forma concisa aquellos elementos susceptibles de ser observados en los videojuegos bélicos de manera independiente. Tras un primer estudio piloto, se seleccionaron 28 variables en la versión final del libro de códigos.

En segundo lugar, se efectuó la elección de la muestra. Dado que el objetivo de la presente investigación es estudiar el contenido representacional de los videojuegos y no solo sus aspectos lúdicos o puramente narrativos, en lugar de la habitual distinción en género de aventuras, rol, disparo en primera persona, etc., se optó por agrupar todos estos géneros como "géneros bélicos" y considerar un videojuego como perteneciente a esta clasificación a partir de los criterios propuestos por Eberwein (2010, p. 45) para el género cinematográfico, de modo que estos son a) narrativa centrada directamente en episodios del conflicto (batallas, preparación, escenario inmediatamente posterior), b) la participación activa de los protagonistas durante el conflicto y c) el efecto de la guerra en las relaciones entre los personajes. Esta agrupación, además, se justifica gracias a los evidentes vínculos de esta clase de ocio audiovisual con otros sectores más tradicionales de la industria del entretenimiento, especialmente el cine bélico estadounidense como referente cultural y generador de contenidos que ha servido de modelo para el desarrollo de sagas de videojuegos como Call of Duty, caracterizada por sus escenarios espectaculares y sus largas cinemáticas inspiradas en películas bélicas (Ramsey, 2015). Teniendo en cuenta estos criterios, se seleccionaron todos aquellos videojuegos que se encontrasen entre los diez más vendidos mensualmente en España, o bien entre los diez más descargados mensualmente en las principales plataformas gratuitas de Steam, Apple Store y Google Play entre 2005 y 2015, según los datos reflejados en la web de la Asociación Española de Videojuegos (2016) y la Interactive Software Federation of Europe (2016), respectivamente. De esta forma, se obtuvo 62 videojuegos bélicos (cuyo listado puede consultarse si se desea en el anexo 1). A continuación, se extrajo de cada uno de ellos un 
gameplay (es decir, una grabación en vídeo de una partida real) de una fase o escenario del videojuego aleatoria de $15 \mathrm{~min}$, que conformó una muestra final de 15,5 h de material audiovisual de análisis.

En tercer lugar, se procedió a la codificación de la muestra empleando a un equipo de siete codificadores independientes, previamente entrenados en la aplicación del libro de códigos y hojas de respuesta. Todos los codificadores analizaron el total de la muestra, tras lo cual se obtuvo una fiabilidad interjueces mediante el alfa de Krippendorff de $\mathrm{M}=0,73(\mathrm{dt}=0,14)$, de modo que la más baja es de 0,68 y la más alta es de 0,91. Tres variables fueron eliminadas del análisis final por poseer un $\alpha \leq 0,66$, según las recomendaciones de Krippendorff (2004).

\section{Resultados}

En lo relativo al discurso legitimador del conflicto a través de la ambientación, los datos del análisis de contenido reflejan que, a la hora de describir el escenario donde transcurre el videojuego, en el 52,5\% de los casos se emplea un enmarque de legítima defensa por parte de la facción a la que pertenece el jugador, frente al $31,1 \%$ en el que es el bando del protagonista quien toma la iniciativa para iniciar el conflicto y el $16,4 \%$ en el que no existe un discurso establecido o es poco conciso. La conquista de recursos o territorios como justificador del conflicto, a su vez, se observa en el 30,6\% de los videojuegos, mientras que en el $21 \%$ su control solo aporta ciertas ventajas al jugador, y en el $48,4 \%$ restante no interviene como elemento clave en la representación del enfrentamiento. En la presencia de personajes explícitamente representados como civiles no combatientes, en el 80,3\% es inexistente, en el 6,6\% se presentan como personajes enemigos (es decir, que se premia su eliminación) y en el $8,2 \%$ como neutrales (a modo de elementos decorativos del escenario); solo en el 4,9\% de los videojuegos el jugador sufre una penalización por atacarlos (figura 1).

Respecto del realismo, el 54,8 \% de los videojuegos analizados están enmarcados en un conflicto verosímil o histórico, frente a un $45,2 \%$ que tienen lugar en universos de fantasía y ciencia ficción. En concreto, en el $22,6 \%$ de los casos, la facción enemiga es un régimen totalitario real (p. ej., 
Alemania nazi), seguido de un $21 \%$ en el que se trata de una organización terrorista o paramilitar y un 19,4\% de una especie no humana (p. ej., extraterrestres); el porcentaje restante pertenece a naciones fantásticas medievales $(16,1 \%)$, potencia no occidental $(9,7 \%)$, una civilización o nación histórica $(8,1 \%)$, u otra facción ficticia indeterminada (3,1\%). A su vez, el 82,3\% de los videojuegos poseen una pretensión realista, mientras que el 17,7 \% tienen una estética cómica o caricaturesca similar a los dibujos animados. Además, en el 16,1 \% de la muestra, la eliminación de los enemigos o el logro de objetivos va acompañado de un efecto sonoro irreal, como una breve melodía desenfadada, sonido de monedas o aplausos, a diferencia de un $83,9 \%$ en el que no se observa esta clase de elementos sonoros decorativos.

\section{Figura 1. Discurso legitimador}

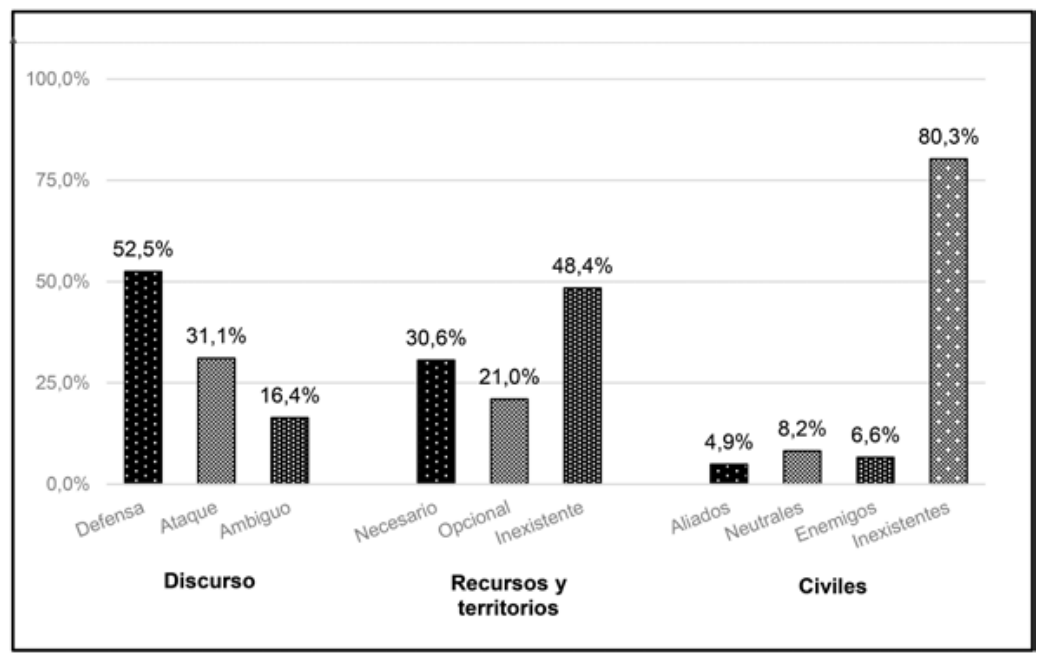

Fuente: elaboración propia.

En la deshumanización de los enemigos, los elementos relacionados con los atributos físicos pueden observarse, por un lado, en función de la naturaleza de los soldados adversarios, los cuales son humanos en el 67,2\% de los videojuegos, humanoides en el 29,5\% y solo monstruos no antropomórficos en el 3,3\%. Por otro lado, a través de su apariencia, de modo que esta es homogénea en un $85,5 \%$ de la muestra al poseer los combatientes enemigos un uniforme, un esquema de color o una simbología compartida que hace difícil distinguirlos entre sí, frente a una apariencia variada e 
individualizadora en el 14,5\% de los casos. En la misma línea, el 61,3\% de los personajes rivales tiene el rostro tapado o no visible, o bien debido a que portan cascos o máscaras, o bien por su tamaño y posición respecto del punto de vista del jugador, mientras que en el 38,7 \% restante sí pueden observarse sus rasgos, expresión facial, etc. (figura 2).

\section{Figura 2. Atributos físicos de los enemigos}

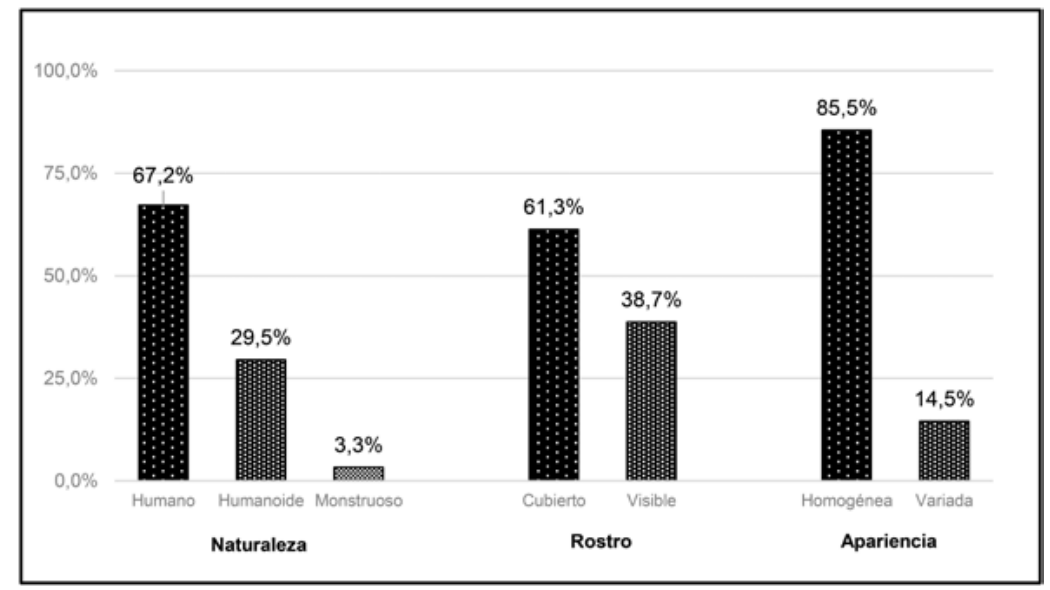

Fuente: elaboración propia.

El comportamiento de los soldados de la facción contraria, por otra parte, es autónomo e independiente de sus compañeros en el 80,6 \% de los videojuegos, de manera que solo el 19,4 \% se comportan de forma gregaria y colectiva. Del mismo modo, el 91,9\% de los enemigos son presentados como seres inteligentes y dueños de sus actos, frente al 8,1\% de ellos que se describen como autómatas sin voluntad.

En lo referente al papel que desempeñan los enemigos en el desarrollo de una partida, en el $79 \%$ de los videojuegos enfrentarse a los soldados rivales es la actividad principal del juego, a diferencia de un $21 \%$ en el que enfrentarse a los adversarios no es necesariamente el centro en torno al cual gira el conflicto armado. Además, los combatientes contrarios nunca muestran comportamientos de rendición en la práctica totalidad de los casos $(98,4 \%)$, por lo que se observa solo en un único videojuego enemigos que renuncian a combatir cuando se encuentran en desventaja o aco- 
rralados (1,6\%). Respecto de la representación del daño causado, el 85,5\% de los enemigos emiten sonidos de dolor o de lesiones físicas al ser atacados, mientras que en el 14,5\% permanecen en silencio o no reaccionan al sufrir daño. El uso de eufemismos para describir la eliminación de los soldados es, por otro lado, minoritario, al estar presente en el 3,2 \% de la muestra; en el 96,8\% restante, se emplean explícitamente términos como muerte o asesinato o elementos visuales como lápidas, fantasmas o cadáveres. En el 72,6 \% de los videojuegos, los soldados abatidos permanecen como un elemento decorativo más del escenario hasta el final de la partida, mientras que en el 27,4 \% desaparecen al cabo de unos segundos.

Prestando atención a los elementos vinculados a la desindividualización del jugador, en cuanto a la construcción del avatar, el análisis de contenido refleja que en el 85,5\% de los casos los personajes están diseñados por defecto y no se permite al usuario decidir sobre su apariencia física, su género o aspectos de su identidad (nombre, facción, etc.), frente a un 14,5\% en el que sí se permite personalizar al protagonista del videojuego. Por otro lado, en el 59,7 \% el personaje protagonista tiene un historia o trasfondo conocido, en que se explicita su nombre propio, sus orígenes o motivaciones para participar activamente en el conflicto, en contraste con el 40,3\% de los casos en el que el jugador encarna a combatientes anónimos (figura 3 ).

\section{Figura 3. Construcción del avatar del jugador}

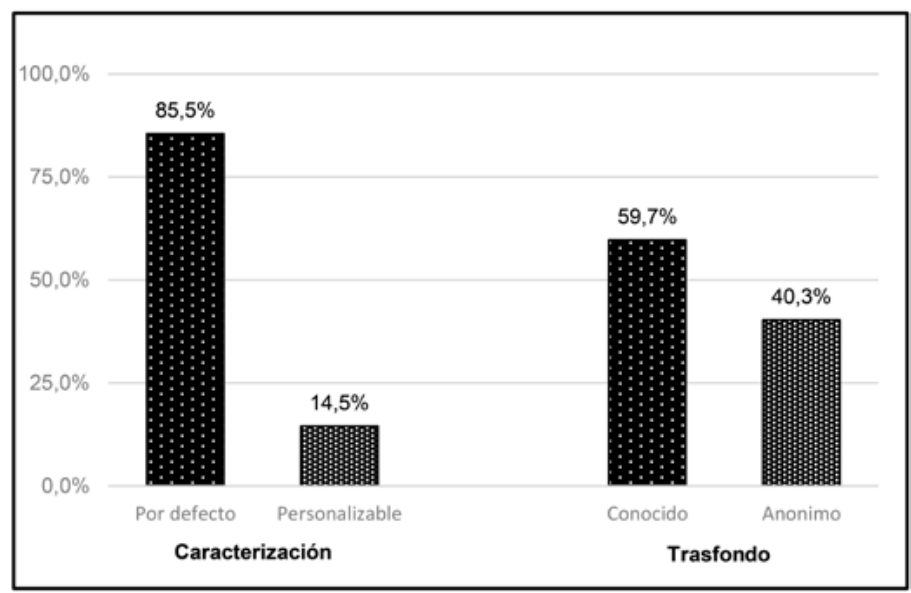

Fuente: elaboración propia. 
Por último, respecto de la representación de las consecuencias de participar en un enfrentamiento, puede observarse que en el $82,3 \%$ de los videojuegos el jugador maneja a un único personaje, de manera que al ser este abatido la partida se interrumpe temporal o definitivamente y obliga al usuario a comenzar de nuevo desde un determinado punto de la trama o el escenario; en cambio, en el 17,7 \% de los videojuegos, el jugador controla varios personajes a la vez, de tal forma que la pérdida de uno o varios de ellos no supone una derrota como tal, sino una desventaja en relación con el adversario. Cuando el personaje o personajes protagonistas son alcanzados por las armas adversarias, en el 62,9\% de los casos se informa al usuario de que se está recibiendo daños mediante alarmas sonoras o visuales, mientras que en el $37,1 \%$ restante no se emite ningún tipo de aviso reconocible. Además, solo en el 4,8 \% de los videojuegos el avatar controlado por el jugador sufre algún tipo de incapacidad cuando es atacado (como ralentización de movimientos, reducción de visión o pérdida de habilidades) frente al 95,2 \% en el que se muestra totalmente invulnerable hasta ser derrotado definitivamente. En la misma línea, los personajes pueden recuperar sus puntos de salud de manera inmediata o progresiva en el 55,9\% y el 18,6 \% de los casos, respectivamente, mediante el uso de objetos (pociones, botiquines) o situándose en determinadas zonas del escenario, incluso si se encuentran en medio de un enfrentamiento; por el contrario, el $25,4 \%$ restante no puede recuperar su resistencia inicial, salvo que sean derrotados o la partida finalice. En cuanto a la inmersión en la acción, en el $45,2 \%$ de los videojuegos, se emplea un punto de vista en primera persona, en el 32,3\% en tercera persona (es decir, la cámara se sitúa a media distancia alrededor del personaje controlado por el usuario de manera que aparece en la pantalla en todo momento) y en el $22,6 \%$ desde una perspectiva panorámica en la que grandes grupos de personajes son visibles simultáneamente.

\section{Discusión}

Como ha podido observarse, existen numerosos elementos legitimadores, deshumanizadores y desindividualizadores que se manifiestan de manera muy recurrente en los videojuegos analizados. En primer lugar, es común que la ambientación de los videojuegos bélicos se plantee desde un discurso de defensa ante un enemigo agresivo, demonizado, implacable y que no 
se rinde jamás, que solo busca la destrucción de la facción a la que pertenece el jugador, sin hacer hincapié en el papel que el control de los recursos o territorios puede desempeñar en las razones reales que llevan al conflicto o invisibilizando la presencia de civiles durante los enfrentamientos. De este modo, la guerra se representa como un mero combate entre ejércitos rivales cuyo único objetivo es acabar violentamente con su adversario, lo que reduce toda posible interacción a una lógica de "ellos o nosotros". A su vez, los soldados de la facción contraria se representan de una manera impersonal y poco individualista, predominantemente con el rostro tapado y portando todos el mismo uniforme y simbología que los hace difíciles de distinguir entre sí, de manera que para el jugador sean solo enemigos anónimos y reemplazables. En la misma línea, en la mayoría de las ocasiones el usuario no puede personalizar un avatar propio, sino que controla uno o varios personajes prediseñados que, sin embargo, y en contraste también con lo que ocurre con los enemigos, sí poseen un trasfondo conocido, una identidad y una historia personal que aporta motivos o razones para participar en el conflicto.

Por otra parte, en un alto porcentaje de casos, los enfrentamientos se pretenden mostrar de una forma realista, adulta y cruda. Lejos de predominar un lenguaje eufemístico o una puesta en escena edulcorada, durante los combates se puede percibir habitualmente el daño causado a los enemigos tanto a través de sus gritos de dolor o sonidos de lesiones como en elementos visuales tales como sangre o enemigos abatidos que se incorporan al escenario como elementos decorativos. Contradictoriamente, el personaje que controla el jugador a menudo se representa como invulnerable a la mayoría de los ataques, de modo que es capaz de seguir combatiendo pese a haber recibido heridas, recuperarse de forma inmediata y solo siendo derrotado tras sufrir una desproporcionada cantidad de daño en comparación con los enemigos con los que combate.

\section{Conclusiones}

En resumen, los datos obtenidos tras el análisis de contenido dibujan un prototipo de videojuego en el que el usuario encarna a un héroe excepcional que debe enfrentarse a hordas de enemigos implacables y sin rostro para 
salvar de la destrucción a su facción, que participa en batallas en las que el principal objetivo es exterminar al oponente. Todo ello ambientado en un escenario con pretensiones realistas y épicas, tanto si se trata de universos reales como de carácter fantástico. Como es obvio, este prototipo no es más que eso, un sumario de los elementos más comunes en los videojuegos analizados, pero ello no implica que dichos elementos se den siempre de manera conjunta en todos los casos. El análisis de la distribución de frecuencias realizado no permite definir diferentes perfiles de videojuegos, pues para ello sería necesario extender el estudio al análisis de contingencias o a la elaboración de clústeres de las variables que componen esta investigación, trabajo metodológico que excede los objetivos propuestos para este artículo y que se reserva para posteriores trabajos. Sería, además, recomendable ampliar el análisis de contenido a nuevas variables que profundicen de manera más exhaustiva en los ejes teóricos planteados, y extender el periodo de muestreo de los videojuegos seleccionados a los años posteriores a 2015, dada la naturaleza en constante cambio de esta clase de ocio audiovisual.

En la valoración de resultados, tampoco se pretende afirmar que esta clase de elementos representacionales no puedan variar su presencia dentro de un mismo videojuego, pues es necesario recordar que en la metodología del análisis de contenido, a diferencia de un estudio de caso, solo se ha analizado un fragmento al azar en forma de gameplay, el cual puede coincidir o no con la tónica general de la obra audiovisual. Por ejemplo, en la saga de juegos Metal Gear Solid, es común encontrar de forma predominante elementos ludológicos que invitan al jugador a no emplear la violencia contra los enemigos; sin embargo, su uso sí se contempla de forma eventual en determinadas ocasiones, y es precisamente la forma de representar dicha violencia (sea recurrente o anecdótica) lo que esta investigación pretende abordar. También cabe destacar, una vez más, que el presente estudio no posee como objetivo analizar en profundidad la narrativa y su interacción con los elementos ludológicos que hacen único a un videojuego, sino identificar los puntos en común entre las obras audiovisuales de este género. Sin duda, esta clase de estudios posee gran interés para la comunidad científica, pero de nuevo sobrepasa el diseño original de esta investigación y los objetivos planteados por los autores; la intención del presente 
trabajo es servir como marco de referencia que ayude a contextualizar futuros estudios de caso, así como investigaciones experimentales centradas en los efectos mediáticos de los videojuegos bélicos, especialmente desde la perspectiva de la teoría de la comunicación.

No obstante, este primer acercamiento descriptivo sí permite asentar una base empírica que refleje la recurrente presencia o ausencia de determinadas representaciones sobre la guerra y sus participantes en los videojuegos y que justifique, a su vez, el estudio de su posible influencia en los jugadores. Por ejemplo, el hecho de que la mayoría de los videojuegos analizados esté ambientada en escenarios verosímiles y cercanos históricamente hablando, tales como la Segunda Guerra Mundial o la llamada guerra contra el terror, unido a la predominancia de determinados elementos legitimadores del conflicto (como el discurso de defensa o la deshumanización del enemigo), refuerza la idea planteada por autores como Chapman (2016), Ford (2016), Gagnon (2010), Kolek \& Šisler (2017), Lizardi (2009), Santorineos \& DimitriadiSantorineos et. al. (2006) o, Williams (2006), de que los videojuegos pueden ser una potente herramienta de cambio de actitudes hacia conflictos bélicos reales. En última instancia, este cambio de actitud puede derivar en una legitimación de determinadas posturas políticas y de intervención militar en contiendas actuales como los enfrentamientos en Oriente Medio o las tensiones con potencias no occidentales; del mismo modo, los videojuegos también pueden convertirse fácilmente en perpetuadores de prejuicios negativos hacia determinados colectivos, como los combatientes de origen árabe que demasiado a menudo encarnan el papel de antagonistas (Šisler, 2008).

Además, y aunque como ya se ha mencionado en los videojuegos predomina una estética realista y adulta, la minimización de los daños a la población civil y la aparente invulnerabilidad del héroe protagonista pueden llevar a una visión idealizada o banal de la guerra. En un mundo en el que el desarrollo de nuevas tecnologías militares, como los drones de combate o el control telemático de los armamentos, hacen que la guerra se parezca cada vez más a un videojuego, estudiar los mecanismos psicológicos que existen en esta clase de ocio y que hacen posible su disfrute a través de la 
desconexión moral (Hartmann \& Vorderer, Hartmann et. al., 2010; Klimmt et al., 2006) puede ayudar a entender, a su vez, los retos y dilemas éticos a los que los soldados deben enfrentarse hoy en día.

En definitiva, son muchas las líneas aún abiertas en el estudio de los videojuegos y la influencia de su consumo en los jugadores y, en consecuencia, es necesaria una labor investigadora constante y conjunta de las ciencias sociales en la recopilación de una base empírica sólida y extensa que respalde las conclusiones obtenidas. A este efecto, la presente investigación pretende ser un modesto aporte más que sirva de punto de partida para futuros trabajos en el campo de los game studies, especialmente en el contexto español en el que los videojuegos cada vez adquieren más relevancia en la sociedad de la información.

\section{Referencias}

Anderson, C. A. \& Dill, K. E. (2000). Video games and aggressive thoughts, feelings, and behavior in the laboratory and in life. Journal of Personality and Social Psychology, 78(4), 772-790. DOI: https://doi. org/10.1037/0022-3514.78.4.772

Anderson, C. A. (2004). An update on the effects of playing violent video games. Journal of Adolescence, 27(1), 113-122. DOI: https://doi. org/10.1016/j.adolescence.2003.10.009

Asociación Española de Videojuegos. (2016, marzo). Los videojuegos más vendidos. Recuperado de Videojuegos: http://www.aevi.org.es/laindustria-del-videojuego/los-videojuegos-mas-vendidos/

Bandura, A. (1999). Moral disengagement in the perpetration of inhumanities. Personality and Social Psychology Review, 3(3), 193-209. DOI: https://doi.org/10.1207/s15327957pspr0303_3

Bar-Tal, D. (1989). Delegitimization: The extreme case of stereotyping and prejudice. En D. Bar-Tal, C. F. Graumann, A. W. Kruglanski \& W. 
Stroebe (Eds.), Stereotyping and prejudice (pp. 169-182). Nueva York, EE. UU.: Springer.

Bellmunt, M. (2012). Player 1 kills player 2: representación de la violencia en el videojuego. RUTA: revista universitària de treballs acadèmics, 4, 1-12. Recuperado de https://ddd.uab.cat/pub/ruta/ruta_ a2012n4/ruta_a2012n4a4.pdf

Bensley, L. \& Van Eenwyk, J. (2001). Video games and real-life aggression: Review of the literature. Journal of Adolescent Health, 29(4), 244257. DOI: https://doi.org/10.1016/s1054-139x(01)00239-7

Chapman, A. (2016). It's hard to play in the trenches: World War I, collective memory and videogames. Game Studies, 16(2). Recuperado de http://gamestudies.org/1602/articles/chapman

Diener, E. (1976). Effects of prior destructive behavior, anonymity, and group presence on deindividuation and aggression. Journal of Personality and Social Psychology, 33(5), 497-507. DOI: https://doi. org/10.1037/0022-3514.33.5.497

Dill, K. E. \& Dill, J. C. (1999). Video game violence: A review of the empirical literature. Aggression and Violent Behavior, 3(4), 407-428. DOI: https://doi.org/10.1016/S1359-1789(97)00001-3

Eberwein, R. (2009). The Hollywood war film. Malden, EE. UU.: John Wiley \& Sons.

Ferguson, C. J. \& Kilburn, J. (2010). Much ado about nothing. The misestimation and overinterpretation of violent video game effects in Eastern and Western nations: Comment on Anderson et al. (2010). Psychological Bulletin, 136(2), 174-178. DOI: https://doi. org/10.1037/a0018566

Ford, D. (2016). "eXplore, eXpand, eXploit, eXterminate": Affective writing of postcolonial history and education in Civilization V. Game 
Studies, 16(2). Retrieved from http://gamestudies.org/1602/articles/ford

Gagnon, F. (2010). "Invading Your Hearts and Minds”: Call of Duty ${ }^{\circledR}$ and the (Re) Writing of Militarism in US Digital Games and Popular Culture. European Journal of American Studies, 5(5-3). DOI: https://doi.org/10.4000/ejas.8831

Gerbner, G. \& Gross, L. (1976). Living with television: The violence profile. Journal of Communication, 26(2), 172-199. DOI: https://doi. org/10.1111/j.1460-2466.1976.tb01397.x

Griffiths, M. (1999). Violent video games and aggression: A review of the literature. Aggression and Violent Behavior, 4(2), 203-212. DOI: https://doi.org/10.1016/s1359-1789(97)00055-4

Hartmann, T. \& Vorderer, P. (2010). It's okay to shoot a character: Moral disengagement in violent video games. Journal of Communication, 60(1), 94-119. DOI: https://doi.org/10.1111/ j.1460-2466.2009.01459.x

Haslam, S. A. y Reicher, S. D. (2004). Visión crítica de la explicación de la tiranía basada en los roles: pensando más allá del Experimento de la Prisión de Stanford. Revista de Psicología Social, 19(2), 115-122. DOI: https://doi.org/10.1174/021347404773820936

Haslam, N. (2006). Dehumanization: An integrative review. Personality and Social Psychology Review, 10(3), 252-264. DOI: https://doi. org/10.1207/s15327957pspr1003_4

Hess, A. (2007). "You Don't Play, You Volunteer”: Narrative Public Memory Construction in Medal of Honor: Rising Sun. Critical Studies in Media Communication, 24(4), 339-356. DOI: https://doi. org/10.1080/07393180701567729 
Igartua, J. J. y Páez, D. (1997). El cine sobre la Guerra Civil Española: una investigación sobre su impacto en actitudes y creencias. Boletín de Psicología, 57, 7-39. Recuperado de http://diarium.usal.es/jigartua/files/2012/07/Igartua-Paez_Films-SCW_Boletin-de-Psicologia_1997.pdf

Interactive Software Federation of Europe. (2016, noviembre 1). Industry facts. Recuperado de https://www.isfe.eu/industry-facts

Klimmt, C., Schmid, H., Nosper, A., Hartmann, T. \& Vorderer, P. (2006). How players manage moral concerns to make video game violence enjoyable. Communications, 31(3), 309-328. DOI: https://doi. org/10.1515/COMMUN.2006.020

Kolek, L. \& Šisler, V. (2017). Representation of history in computer games and attitude change: empirical study design. En ECGBL 2017: 11th European Conference on Games Based Learning (pp. 829-834). Academic Conferences International Limited.

Krippendorff, K. (2004). Reliability in content analysis: Some common misconceptions and recommendations. Human Communication Research, 30(3), 411-433. DOI: https://doi. org/10.1111/j.1468-2958.2004.tb00738.x

Leyens, J. P., Rodriguez-Perez, A., Rodriguez-Torres, R., Gaunt, R., Paladino, M. P., Vaes, J. \& Demoulin, S. (2001). Psychological essentialism and the differential attribution of uniquely human emotions to ingroups and outgroups. European Journal of Social Psychology, 31(4), 395-411. doi: https://doi.org/10.1002/ejsp.50

Lizardi, R. (2009). Repelling the invasion of the "Other": Post-apocalyptic alien shooter videogames addressing contemporary cultural attitudes. Eludamos. Journal for Computer Game Culture, 3(2), 295-308. Retrieved from https://www.eludamos.org/index.php/eludamos/ article/view/vol3no2-11 
Marcano Lárez, B. E. (2014). Factores emocionales en el diseño y la ejecución de videojuegos y su valor formativo en la sociedad digital: el caso de los videojuegos bélicos. Salamanca, España: Universidad de Salamanca.

Miguel Pascual, R. de (2012). Cuestiones en torno al poderoso efecto de los videojuegos violentos: del neoconductismo a la cognición social. ICONO 14, 4(1), 176-204. DOI: https://doi.org/10.7195/ ri14.v4i1.403

Olson, C. K. (2004). Media violence research and youth violence data: Why do they conflict? Academic Psychiatry, 28(2), 144-150. DOI: https://doi.org/10.1176/appi.ap.28.2.144

Ouellette, M. A. (2008). I hope you never see another day like this': Pedagogy \& allegory in 'post 9/11'video games. Game Studies, 8(1). Retrieved from http://gamestudies.org/0801/articles/ouellette_m

Postmes, T. \& Spears, R. (1998). Deindividuation and antinormative behavior: A meta-analysis. Psychological bulletin, 123(3), 238. DOI: https://doi.org/10.1037/0033-2909.123.3.238

Pötzsch, H. \& Hammond, P. (2016). War/game: Studying relations between violent conflict, games, and play. Game Studies, 16(2). Retrieved from http://gamestudies.org/1602/articles/potzschhammond

Power, M. (2007). Digitized virtuosity: Video war games and post-9/11 cyber-deterrence. Security Dialogue, 38(2), 271-288. DOI: https:// doi.org/10.1177/0967010607078552

Ramsay, D. (2015). Brutal Games: Call of Duty and the Cultural Narrative of World War II. Cinema Journal, 54(2), 94-113. DOI: https:// doi.org/10.1353/cj.2015.0015

Rehm, J., Steinleitner, M. \& Lilli, W. (1987). Wearing uniforms and aggression-A field experiment. European Journal of Social Psychology, 17(3), 357-360. DOI: https://doi.org/10.1002/ejsp.2420170310 
Rodríguez Serrano, A. (2014). Cuando los videojuegos escribieron el Holocausto: análisis de Wolfenstein: The New Order (Machinegames, 2014). Historia y Comunicación Social, 19, 193-207. DOI: https:// doi.org/10.5209/rev_hics.2014.v19.47292

Saleem, M. \& Anderson, C. A. (2013). Arabs as terrorists: Effects of stereotypes within violent contexts on attitudes, perceptions, and affect. Psychology of Violence, 3(1), 84. DOI: https://doi.org/10.1037/ a0030038

Sample, M. L. (2008). Virtual torture: Video games and the war on terror. Game Studies, 8(2). Retrieved from http://gamestudies.org/0802/ articles/sample

Santorineos, M. \& Dimitriadi, N. (2006). Gaming realities: A challenge for digital cultures. Athens, Greece: Fournos.

Silke, A. (2003). Deindividuation, anonymity, and violence: Findings from Northern Ireland. The Journal of Social Psychology, 143(4), 493-499. DOI: https://doi.org/10.1080/00224540309598458

Šisler, V. (2008). Digital Arabs: Representation in video games. European Journal of Cultural Studies, 11(2), 203-220. DOI: https://doi. org/10.1177/1367549407088333

Stamenković, D., Jaćević, M. \& Wildfeuer, J. (2017). The persuasive aims of Metal Gear Solid: A discourse theoretical approach to the study of argumentation in video games. Discourse, Context \& Media, 15, 11-23. DOI: https://doi.org/10.1016/j.dcm.2016.12.002

Struch, N. \& Schwartz, S. H. (1989). Intergroup aggression: Its predictors and distinctness from in-group bias. Journal of Personality and Social Psychology, 56(3), 364-373. DOI: https://doi.org/10.1037/00223514.56.3.364 
Van Mierlo, J. \& Van den Bulck, J. (2004). Benchmarking the cultivation approach to video game effects: A comparison of the correlates of TV viewing and game play. Journal of Adolescence, 27(1), 97-111. DOI: https://doi.org/10.1016/j.adolescence.2003.10.008

Voorhees, G. (2014). Play and possibility in the rhetoric of the war on terror: The structure of agency in Halo 2. Game Studies, 14(1). http://gamestudies.org/0802/articles/gvoorhees

Williams, D. (2006). Virtual cultivation: Online worlds, offline perceptions. Journal of Communication, 56(1), 69-87. DOI: https://doi. org/10.1111/j.1460-2466.2006.00004.x

Zimbardo, P. G. (1970). The human choice: Individuation, reason and order versus deindividuation, impulse and chaos. En W. J Arnold \& D. Levine (Eds.), Nebraska symposium on motivation, 1969. Lincoln, EE. UU.: University of Nebraska Press.

Zimbardo, P. (2008). El efecto Lucifer: el porqué de la maldad. Barcelona, España: Paidós. 


\section{Anexo 1. Videojuegos analizados}

Age of Empires II: HD Edition (Ensemble Studios, 2013)

Age of Empires III (Ensemble Studios, 2005)

Arma III (bohemia Interactive, 2013)

Army of Two (EA, 2013)

Battlefield 3 (EA Digital Illusions, 2011)

Battlefield 4 (EA Digital Illusions, 2013)

Boom Beach (Supercell, 2014)

Call of Duty 2 (Infinity Ward, 2005)

Call of Duty: Advanced Warfare (Sledgehammer Games, 2014)

Call of Duty: Black Ops (Treyarch, 2010)

Call of Duty: Black Ops II (Treyarch, 2012)

Call of Duty: Ghosts (Infinity Ward, 2013)

Call of Duty: Modern Warfare 2 (Infinity Ward, 2009)

Call of Duty: Modern Warfare 3 (Infinity Ward, 2011)

Call of Duty: World at War (Treyarch, 2009)

Chivalry: Medieval Warfare (Torn Banner Studios, 2012)

Civilization V (Firaxis Games, 2010)

Clash of Clans (Supercell, 2012)

Commandos Strike Force (Pyro Studios, 2006)

Company of Heroes (Relic Entertainment, 2006)

Counter Strike: Global Offensive (Valve, 2013)

Crysis 2 (Crytek, 2011)

Defense of the Ancients 2 (Valve, 2013)

Empire: Total War (The Creative Assembly, 2009)

Far Cry 4 (Ubisoft, 2014)

Gears of Wars 3 (Epic Games, 2011)

Gears of Wars: Judgment (Epic Games, 2013)

Gears of Wars: Ultimate Edition (Epic Games, 2006)

Guild Wars (ArenaNet, 2005)

Guild Wars 2 (ArenaNet, 2012)

Halo 3 (Bungie Studios, 2007)

Halo 3: ODST (Bungie Studios, 2009)

Halo: Reach (Bungie Studios, 2010) 
Heroes \& Generals (Reto-Moto, 2014)

Imperium III: Las grandes batallas de Roma (Haemimont Games, 2005)

Insurgency (New World Interactive, 2014)

Just Cause 3 (Avalanche Studios, 2015)

Killzone 2 (Guerrilla Games, 2009)

Killzone 3 (Guerrilla Games, 2011)

Mass Effect 3 (BioWare, 2012)

Medal of Honor (EA, 2012)

Medal of Honor: European Assault (EA, 2005)

Mercenaries 2: World in Flames (Pandemic Studios, 2008)

Metal Gear Rising: Revengeance (Platinum Games, 2013)

Metal Gear Solid 4: Guns of the Patriots (Kojima Productions, 2008)

Metal Gear Solid V: Ground Zeroes (Kojima Productions, 2014)

Metal Gear Solid V: The Phantom Pain Day (Kojima Productions, 2015)

PlanetSide 2 (Daybreak Game Company, 2012)

Resistance: Fall of Man (Insomniac Games, 2006)

Señor de los Anillos: La Batalla por la Tierra Media II (EA, 2006)

Sniper Elite V2 (Rebellion Developments, 2012)

Star Wars: Battlefront 2 (Pandemic Studios, 2005)

Starcraft II: Wings of Liberty (Blizzard Entertainment, 2014)

Team Fortress 2 (Valve, 2007)

War Thunder (Gaijin Entertainment, 2012)

Warframe (Digital Extremes, 2013)

Warhammer 40.000 Dawn of War II (Relic Entertainment, 2011)

Warhammer Online: Age of Reckoning (EA Mythic, 2008)

World of Warcraft (Blizzard Entertainment, 2005)

World of Warcraft: Burning Crussade (Blizzard Entertainment, 2007)

World of Warcraft: Cataclysm (Blizzard Entertainment, 2010)

World of Warcraft: Mists of Pandaria (Blizzard Entertainment, 2012) 